\title{
BMJ Open Barriers-enablers-ownership approach: a mixed methods analysis of a social intervention to improve surgical antibiotic prescribing in hospitals
}

\author{
Jennifer Broom (D) , ${ }^{1,2}$ Alex Broom, ${ }^{3}$ Chris Anstey, ${ }^{2,4}$ Katherine Kenny (D) , ${ }^{3}$ \\ Sharon Young, ${ }^{5}$ David Grieve, ${ }^{4,5}$ David Sowden, ${ }^{5}$ Aishwarya Jangam, ${ }^{6}$ \\ Andrew Henderson, ${ }^{7,8}$ Alexandra Melon, ${ }^{9}$ Renee Tabone, ${ }^{2,4,6}$ Drew Farquhar, ${ }^{9}$ \\ Henry Harding, ${ }^{4}$ Seyed Ehsan Panahi, ${ }^{9}$ Tyler Chin, ${ }^{5}$ Mariya Abdullah, ${ }^{9}$ \\ Louise Waterhouse, ${ }^{5}$ Clarissa Lo, ${ }^{5,6}$ Rhiannon Parker, ${ }^{3}$ The Lan Bui, ${ }^{5}$ \\ Marianne C Wallis (D) ${ }^{10}$
}

To cite: Broom J, Broom A, Anstey C, et al. Barriersenablers-ownership approach: a mixed methods analysis of a social intervention to improve surgical antibiotic prescribing in hospitals. BMJ Open 2021;11:e046685. doi:10.1136/ bmjopen-2020-046685

- Prepublication history and supplemental material for this paper is available online. To view these files, please visit the journal online (http://dx.doi. org/10.1136/bmjopen-2020046685).

Received 06 November 2020 Revised 18 March 2021 Accepted 08 April 2021

\section{D) Check for updates}

(c) Author(s) (or their employer(s)) 2021. Re-use permitted under CC BY-NC. No commercial re-use. See rights and permissions. Published by BMJ.

For numbered affiliations see end of article.

Correspondence to Dr Jennifer Broom; jennifer.broom@health.qld. gov.au

\section{ABSTRACT}

Objectives To assess an intervention for surgical antibiotic prophylaxis (SAP) improvement within surgical teams focused on addressing barriers and fostering enablers and ownership of guideline compliance. Design The Queensland Surgical Antibiotic Prophylaxis (QSAP) study was a multicentre, mixed methods study designed to address barriers and enablers to SAP compliance and facilitate engagement in selfdirected audit/feedback and assess the efficacy of the intervention in improving compliance with SAP guidelines. The implementation was assessed using a 24-month interrupted time series design coupled with a qualitative evaluation.

Setting The study was undertaken at three hospitals (one regional, two metropolitan) in Australia.

Participants SAP-prescribing decisions for 1757 patients undergoing general surgical procedures from three health services were included. Six bimonthly time points, pre-implementation and post implementation of the intervention, were measured. Qualitative interviews were performed with 29 clinical team members. SAP improvements varied across site and time periods. Intervention QSAP embedded ownership of quality improvement in SAP within surgical teams and used known social influences to address barriers to and enablers of optimal SAP prescribing.

Results The site that reported senior surgeon engagement showed steady and consistent improvement in prescribing over 24 months (prestudy and poststudy). Multiple factors, including resource issues, influenced engagement and sites/time points where these were present had no improvement in guideline compliance. Conclusions The barriers-enablers-ownership model shows promise in its ability to facilitate prescribing improvements and could be expanded into other areas of antimicrobial stewardship. Senior ownership was a predictor of success (or failure) of the intervention across sites and time periods. The key role of senior leaders in change leadership indicates the critical need to engage other specialties in the stewardship agenda.
Strengths and limitations of this study

- The study methodology is innovative in that it integrates key known social influences on antimicrobial prescribing in a structured intervention and quantitatively measures outcomes of this intervention within hospitals.

- The methodology provides a model for embedding surgical antibiotic prophylaxis improvement processes within surgical teams and therefore represents a step towards sustainable antimicrobial stewardship.

- Although antimicrobial-prescribing outcomes are reported, patient outcomes (such as surgical site infections) were not included in this study.

- The study is limited by being performed in Australia, and although these influences on prescribing are likely transferable internationally, further studies of this kind of intervention internationally would yield important cross-context data.

- The quasiexperimental study design limits the evaluation of the data as there were no control sites where the intervention was not implemented.

The influence of contextual factors in limiting engagement clearly identifies issues of resource distributions/ inequalities within health systems as limiting antimicrobial optimisation potential.

\section{INTRODUCTION}

Background

The global crisis of antimicrobial resistance $(\mathrm{AMR})^{1}$ is driven in part by excessive antimicrobial use, which is often inconsistent with established clinical guidelines. ${ }^{2}$ As a result of the frequency of surgical procedures, antimicrobial use for surgical antibiotic prophylaxis (SAP) is among the highest volume categories 
of antimicrobial use within health systems, globally. For example, SAP accounts for $11 \%-15 \%$ of antimicrobials prescribed in Australian hospitals; with inappropriate prescribing documented in $30 \%-57 \%$ of surgical operations, depending on the surgical specialty. ${ }^{3}$ Inappropriate SAP prescribing in this context was related to factors such as choice (eg, allergy mismatch or the antimicrobial administered was too broad or too narrow in antimicrobial spectrum), dosing or timing errors, administration of SAP when not required or duration extended beyond clinical guideline recommendations. Prescribing discordance with guidelines in SAP is reported across different contexts. However, the range is broad, with concordance rates of $0.3 \%-84.5 \%$ reported, depending on, among other factors, location of study, specificity of guidelines used, data recorded and types of surgeries reviewed. ${ }^{4-9}$ In the 2016 Australian Surgical National Antimicrobial Prescribing Survey, SAP was appropriate in just under $57 \%$ in general surgery. ${ }^{3}$

\section{Overuse of SAP results in poorer outcomes}

Guideline concordance in SAP is not only a concern for the development of AMR. SAP prescribing that is discordant with guidelines has also been associated with poorer clinical outcomes. For example, in a large meta-analysis, SAP administered more than 120 min before, or after the incision was associated with a higher risk of surgical site infections. $^{10}$

\section{SAP improvement strategies: sustainability}

There have been significant efforts to improve SAP both in Australia and internationally. Demonstrable improvement in guideline concordance has been achieved in several studies. ${ }^{4-811}$ The value of audit/feedback, multidisciplinary pathways and institutional strategies such as decision support systems have been identified as helpful in improving SAP prescribing. ${ }^{12}$ However, external auditing of practice can be costly and labour intensive, and not all clinical contexts have the resources or technology to support the implementation of decision support systems. SAP improvement studies, like other antimicrobial stewardship (AMS) interventions, commonly involve external auditing of team performance by study investigators who are often pharmacists, ${ }^{13}$ AMS teams ${ }^{5}$ or infection control practitioners. ${ }^{4}$ This can be effective but is not likely to be sustainable in the longer term due to the large number of clinical areas in which antimicrobials are used (and therefore the large volume of audit/feedback processes required for optimisation across different specialties) and is certainly not likely to be possible long term in resourcelimited settings. There is an urgent need for strategies that can be embedded within teams to support long-term antimicrobial-prescribing excellence.

\section{Leadership and senior engagement}

Lack of senior surgical engagement is a recognised issue within surgical AMS,${ }^{14}$ despite identification of the crucial importance of their role in its successful implementation. ${ }^{15}$
In a recent international survey of AMS practices in surgery, for example, surgeons were involved in the stewardship team in only $59 \%$ of cases. ${ }^{16}$ This reflects a broader issue of AMS, in which prescribing improvement strategies which are driven outside of specialties (by infectious diseases or microbiologists) ${ }^{17} 18$ are reported to result in disengagement. Embedding quality improvement strategies within, and as driven by, specialties or individual teams is a potential logical step for consistent and sustainable prescribing changes in other areas of AMS; however, there is a lack of robust evidence around what might represent the most effective approaches within surgical teams. This study examined a strategy to increase surgical engagement and examined the dynamics that were associated with the success (or otherwise) of engagement within surgical teams.

\section{Social factors driving overuse and undermining AMS}

As knowledge of antimicrobial prescribing expands, there has been increased attention towards the previously opaque social influences on antimicrobial use and decision-making (including specifically in SAP decisionmaking). These include such issues as the role of hierarchy within surgical teams, concern for adverse patient outcomes and respect for clinical autonomy (meaning that external teams such as infectious diseases services or dedicated AMS teams are limited in their capacity to influence the internal decision-making of a specialty team) ${ }^{19-22}$ Given the critical need for effective AMS strategies, there is an urgent need for interventions addressing identified determinants of practice in antimicrobial prescribing. ${ }^{23}$

Expected outcomes as a result of improvements in SAP prescribing Concordance with clinical guidelines in SAP prescribing has been associated with reduced rates of surgical site infection $^{1024}$ and appropriate use (limited to evidencebased guidelines) would be expected to reduce the volume of antimicrobial use in hospitals which will reduce the pressure for the development of AMR.

This study was designed to assess an intervention which integrated quality improvement in SAP within surgical teams, with facilitated team processes to address barriers, enablers and ownership in SAP-prescribing improvement.

\section{METHODS}

\section{Clinical context}

This study included patients undergoing general surgical procedures at three hospitals over 24 months during 2018-2019; one university-affiliated specialist referral centre with 450 beds (site 1 ), and two secondary referral hospitals with 250 (site 2) and 175 beds (site 3), in Queensland, Australia. Site 1 had an established AMS team with 1.5 full-time equivalent (FTE) clinical pharmacists, 0.5 FTE AMS clinical nurse and 0.6 FTE Infectious Diseases (ID) physician time committed to AMS (for a health service of 700 beds, four hospitals). Sites 2 and 3 had 0.5 and 1.0 FTE AMS pharmacist time, respectively, 
but limited dedicated ID physician AMS time (0.1 FTE nominally each site). Sites were recruited to represent a range of clinical contexts (varying hospital size and location), within a geographic range that was practical for facilitation by the study facilitator. Three of four sites approached agreed to participation. Junior clinical staff rotated on 3-month intervals. Rotation of juniors to different hospitals (annual rotation) occurred between cycles $2 / 3$ and $8 / 9$ at sites 1 and 2 and between cycles $1 / 2$ and $7 / 8$ at site 3 . There was not a regular attendance of the local AMS team at rounds or team meetings at any site.

\section{Study design}

Queensland Surgical Antibiotic Prophylaxis intervention

The study comprised a mixed methods design ${ }^{2526}$ quantitatively assessing an intervention to embed SAP-prescribing improvement within a surgical team by way of an interrupted time series design ${ }^{27}$ combined with qualitative in-depth individual interviews exploring the experience of the intervention by surgeons and pharmacists at each site (figure 1). Detailed information on the Queensland Surgical Antibiotic Prophylaxis (QSAP) intervention is provided (online supplemental appendix 1). For the quantitative arm, the preintervention and postintervention periods were 12 months in length. This study was designed around the following dimensions, based on evidence the current authors and other researchers internationally have identified as shaping SAP.

Barriers: addressing evidence-based barriers (guideline mistrust, lack of visibility/knowledge of guidelines, lack of prioritisation of antibiotic decision-making in the operating theatre). ${ }^{12} 2022$

Enablers: targeting social dynamics within surgical teams which would be expected to increase compliance with guidelines (hierarchical structures, clarity in role delineation) ${ }^{12} 19$ and provide audit/feedback of compliance with guidelines. ${ }^{13}$

Ownership: fostering surgical ownership/leadership of quality improvement in SAP. ${ }^{18} 28$

\section{Audit feedback}

The director of general surgery at each site agreed to lead the quality improvement cycle within their team and nominated surgical team members to perform auditing. The requirement for auditing within the surgical team was a priority to increase guideline knowledge within the team and to facilitate ownership of the study and the study outcomes.

\section{Collaborative introductory meeting: the barriers-enablers- ownership nexus}

A collaborative meeting started the 12-month intervention period at each site. The facilitator (JB) attended a surgical team meeting and a junior surgical team member at each site presented audit data. A facilitated $45-60 \mathrm{~min}$ discussion around the results, areas of discordance with the guidelines, key evidence-based social influences on prescribing decisions (barriers) and the potential for team leadership in fostering change (enablers). Teams were also asked to collaboratively identify goals for change to occur at each site and team members to be responsible for the ongoing data collection (ownership).

\section{Follow-up meetings}

Follow-up meetings (facilitated by JB), structured around the barriers-enablers-ownership model, occurred every 3-4 months at each site (at least three meetings at each site) for 12 months following the collaborative meeting. This was accompanied by real-time, department-specific data on local compliance with guidelines presented by a local team member. Discussion around guideline discordance was facilitated with a focus on consensus building and leadership from senior surgeons. This allowed a combination of discussion about social and behavioural influences on guideline concordance, as well as reflection on actual, local audit data and why (or why not) change was occurring.

\section{Data collection}

To augment the collaborative and follow-up meetings, an online auditing tool was provided to all sites using the Research Electronic Data Capture database system. Patient data were obtained by retrospective review of the operating lists, medical record, anaesthetic notes and prescribing chart (either electronic medical record or paper chart depending on the site), and included demographic data, operative details, risk factors for surgical site infection and the accuracy of SAP prescribing audited against local SAP guidelines (see table 1 for SAP error definitions). The first 50 elective and acute patients of each month were selected from general surgery operating lists every second month for the 24-month study period and charts were reviewed retrospectively. All data entries were checked for accuracy by the lead investigator (JB). Emails to follow-up data collection and to support juniors in learning and interpreting guidelines against which they were auditing were undertaken by the study facilitator.

\section{Statistical analysis}

Variables in the final data set were tested for normality using the Shapiro-Wilk test. Depending on distribution, differences in overall compliance rates across sites were tested using either standard analysis of variance or the Kruskal-Wallis test. Binary comparisons were made using either a two-tailed t-test for independent samples or the Wilcoxon rank-sum test. Finally, the temporal data were analysed as an interrupted time series using per cent compliance as the outcome variable and bimonthly intervals from the start of the study as the time base. To gauge the immediate effect of the intervention, the results were summarised as the preintervention linear regression slope $\left(\beta_{\text {pre }}\right)$, the postintervention linear regression slope $\left(\beta_{\text {post }}\right)$ and the change in the level of the outcome variable in the period immediately following the intervention $(\Delta)$. Serial 


\section{QSAP}

Queensland Surgical Antibiotic

Prophylaxis Intervention

\section{BACKGROUND}

\begin{tabular}{|c|c|c|}
\hline Barriers & Enablers & Dwnership \\
\hline $\begin{array}{l}\text { Addressing evidence-based } \\
\text { barriers }(\mathbf{1 2 , 2 0 , 2 2 )} \\
\text { - guideline mistrust } \\
\text { - lack of visibility/ } \\
\text { knowledge of guidelines, } \\
\text { - lack of prioritisation of } \\
\text { antibiotic decision-making } \\
\text { in the operating theatre } \\
\text { - fear of adverse outcomes } \\
\text { and litigation }\end{array}$ & $\begin{array}{l}\text { Targeting social dynamics } \\
\text { within surgical teams (12, } \\
\text { 19) } \\
\text { - hierarchical structures, } \\
\text { - clarity in role delineation } \\
\text { Audit/feedback to surgical } \\
\text { team of compliance with } \\
\text { guidelines (13) }\end{array}$ & $\begin{array}{l}\text { Fostering surgical } \\
\text { ownership/leadership of } \\
\text { quality improvement in } \\
\text { SAP (18,22,27) } \\
\text { - intra-professional dynamics } \\
\text { and desire for autonomy } \\
\text { within specialist services } \\
\text { mean leadership of change } \\
\text { within (rather than external } \\
\text { to) specialities is likely to be } \\
\text { more effective }\end{array}$ \\
\hline
\end{tabular}

\section{INTERVENTION}

\section{QSAP Fixed components}

1 Director of general surgery agrees to provide leadership in the study and to identify junior staff members willing to collection audit data

2 Audit data is collected by the surgical team at each site prior to the intervention

3 Audit data is presented by the surgical team at each site at the inaugural meeting and each follow-up meeting (engagement) and a facilitated discussion of influences on SAP decision making is led by the study investigator, including:

1. Areas of discordance with the guidelines - including variation between consultants and whether guidelines represent current evidence, and barriers to guideline uptake including fear of adverse outcomes and litegation

2. Strategies to facilitate adherence including guideline visibility and commnunication with anaesthetics, use of hierarchical influence within surgical units

3. Leadership of the project within the unit

4 - Audit results presented by surgical team

- Facilitated discussion around:

1. Ongoing guideline discordance or improvements

2. Factors within teams contributing to discordance or improvements

\section{QSAP Variable components}

As requested by surgical teams during the study

1 Presentation of guidelines on theatre walls (Sites 1 and 3)

2 Anaesthetic engagement (Site 2)

Ownership

Enablers

Ownership

Ownership

Barriers

Enablers

Figure 1 Queensland Surgical Antibiotic Prophylaxis (QSAP) - integrating known influences on surgical antibiotic decisionmaking: a multimodal intervention. SAP, surgical antibiotic prophylaxis

correlation was controlled using a Prais-Winsten correction. To estimate the overall effect of the intervention, a time series regression was performed from the end of the preintervention period to the end of the study period thus including the intervention period. Associated $p$ values were also reported. A power analysis was performed using previously validated simulation methodology ${ }^{29}$ Assuming an effect size of at least 2.0 (expected intervention effect over its SD), equal preintervention and postintervention time periods with both level and trend change for an autoregressive $^{1}$ type model with an autocorrelation range of \pm 0.9 and a statistical significance of 0.05 gave an estimated power of at least 0.92 for an $n=12$ study. Overall fit of the model was also reported $\left(\mathrm{R}^{2}\right)$. Both STATA (V.15.1) and $\mathrm{R}$ were used for the statistical analyses. The level of significance was set at $\alpha<0.05$ throughout. 
Table 1 Decision errors evaluated in QSAP and the potential risks associated with each error

\begin{tabular}{lll}
\hline SAP decision & Criteria for error & Risks/potential adverse outcomes \\
\hline SAP indication & Given when not indicated & AMR/CDI \\
& Not given when indicated & SSI \\
SAP choice & Spectrum too broad & AMR/CDI \\
& Spectrum too narrow & SSI \\
& Allergy mismatch & Allergic reaction to AB \\
SAP dosing error & Dose or frequency too low & SSIAM \\
\hline SAP timing error & Dose or frequency too high & SSI \\
SAP duration error & Not given within 60 min of incision & AMR/CDI \\
\hline Redosing error & Duration greater than local guideline & SSI \\
\hline
\end{tabular}

$A B$, antibiotic; ADR, adverse drug reaction; AMR, antimicrobial resistance; CDI, Clostridium difficile infection; QSAP, Queensland Surgical Antibiotic Prophylaxis; SAP, surgical antibiotic prophylaxis; SSI, surgical site infection.

\section{Qualitative descriptive study arm}

Individual, semistructured interviews

A formal invitation letter and a participant information and consent form were sent electronically to doctors and pharmacists within the general surgical and AMS teams at each site during the last quarter of the intervention period. Participants took part in an individual semistructured interview (performed by a social scientist experienced in qualitative interviews) using an interview guide which was developed to explore perceptions and experiences of SAP use and implementation of the intervention. ${ }^{30}$ Interviews were digitally recorded and transcribed in full. The thematic analysis of the data was driven by a framework approach. ${ }^{31}$ Independent coding of the data was provided initially by members of the research team (JB and $\mathrm{AB}$ ), and was then crosschecked to facilitate the development of themes (JB, AB and $\mathrm{KK})$, moving towards an overall interpretation of the data. $^{31-33}$

\section{Diary of interactions}

A diary of interactions (both formal interactions such as accounts of meetings, and informal such as email and phone communications with the surgical teams) was kept throughout the study by the study facilitator (JB). Interactions with the surgical team were documented in real time.

\section{Patient and public involvement}

While this article comments on the compliance of SAP prescribing and the experiences of teams in improving prescribing, this research programme also more broadly includes patient and public involvement. Patients, carers, as well as stakeholders in legislative and policy and accreditation-related roles are included in the broader research programme.

\section{RESULTS}

In total, 1757 patients undergoing general surgical procedures at the three sites were included. Prescribing compliance data were collected from patients undergoing operations at 12 bimonthly intervals, from 2017 to 2019. Patient demographic and clinical variables from each site are compared in table 2.

\section{SAP administration: discordance with clinical guidelines}

The appropriateness of SAP decisions is documented in table 3. In the combined (all sites) preintervention group, $56.2 \%$ of SAP decisions were consistent with guidelines in all aspects (choice of antibiotic, dose, timing, duration), and in the postintervention group $70.5 \%$ of decisions were correct. The reasons for guideline discordant SAP decisions at each site are listed in table 3. The largest proportion of variance related to SAP administration where SAP was not indicated, dosing and timing errors. For patients in whom SAP was indicated (and was not given), but were on treatment antibiotics, the treatment antibiotics were assessed as to whether they were appropriate for SAP against local guidelines.

\section{Compliance in SAP prescribing over time}

Overall the change in SAP compliance with local guidelines was significantly different between the study sites (see table 4 and figure 2).

Preintervention $\left(\beta_{\text {pre }}\right)$ : A significant rise in compliance was observed at site 1 . Sites 2 and 3 showed a constant compliance rate before the intervention.

Change at intervention $(\Delta)$ : Site 1 demonstrated a significant drop in compliance rate around the time of the intervention while site 2 demonstrated a significant rise in compliance rate. Site 3 showed no change.

Postintervention $\left(\beta_{\text {post }}\right)$ : Site 1 demonstrated a continued significant rise in compliance rate after the intervention 
Table 2 Between-site comparisons of demographic and clinical variables

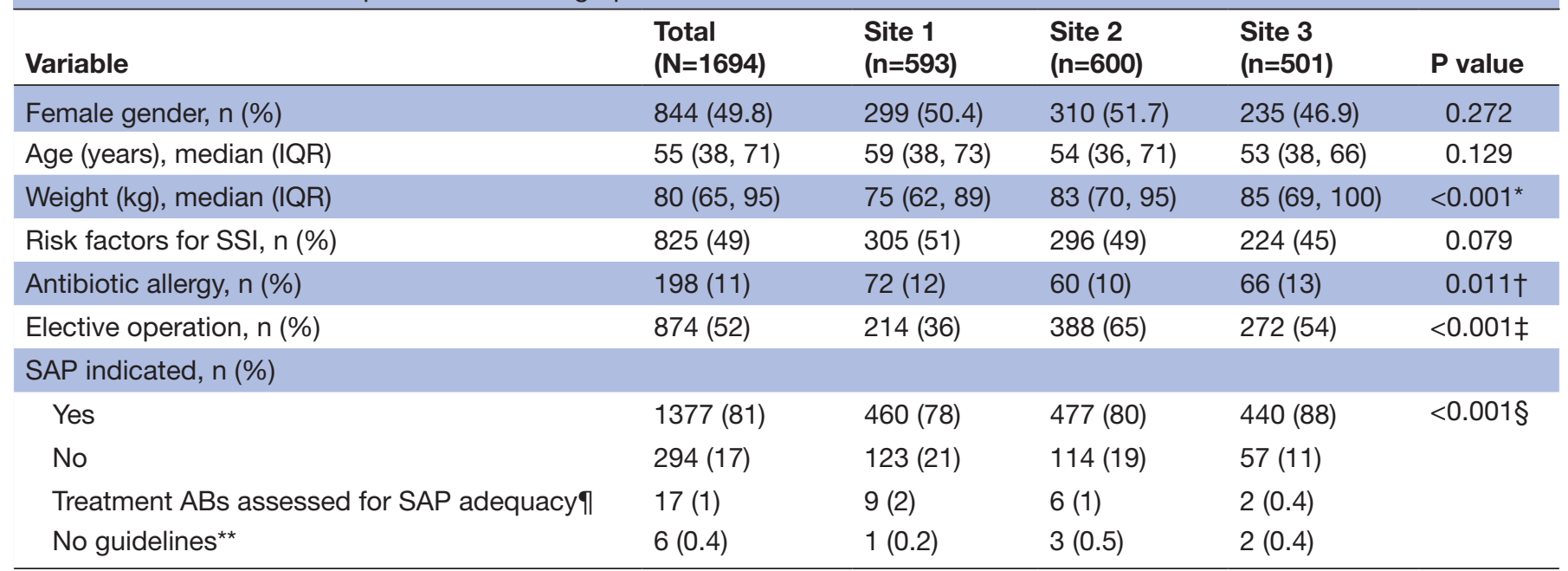

Absolute number $(\mathrm{n})$ and the per cent (based on the number in that group) is represented. $\mathrm{P}$ values $<0.05$ are considered significant. ${ }^{*}$ Patients at sites 2 and 3 were significantly heavier than those at site 1 ( $p<0.001$ in both cases).

†Significantly more allergies reported at site 3 than at site $2(p=0.017)$.

$\ddagger$ All sites were significantly different from one another $(p<0.001)$.

§Pairwise analysis showed significant differences between all sites - site $1 \neq$ site $2 \neq$ site 3 ( $p<0.001$ in each case).

ПWhere patients were receiving treatment antibiotics (and additional SAP was not given), their adequacy for SAP was assessed.

${ }^{* \star}$ The patients for which guidelines were not available for the specific operation type.

$A B$, antibiotic; SAP, surgical antibiotic prophylaxis; SSI, surgical site infection.

though the difference between the two slopes (3.67 vs 5.62) was not significant $(\mathrm{p}=0.105)$. Site 2 demonstrated an initial small non-significant rise followed by a tailing off towards the end of the study period. Site 3 demonstrated an initial rise in compliance rate which fell only rising again after a visit from the study facilitator (see diary of interactions and figure 2).

The cumulative effect was a significant rise in the preintervention period, followed by a non-significant change at the intervention finally leading to a continued significant postintervention rise. The before/after slope comparison (1.51 vs 1.56$)$ was not significant $(\mathrm{p}=0.954)$.

Overall change: Sites 1 and 3 demonstrated a sustained and significant rise in compliance rate from the end of the preintervention period to the end of the study period (3.89 and 4.21 percentage, respectively). Site 2 demonstrated a non-significant change in percentage per time period. Across all sites, there was a significant increase in compliance rate of 2.22 percentage per bimonthly time period.

\section{Qualitative descriptive results \\ Diary record of interactions}

Site 1: An initial delay in starting data collection at site 1 was resolved by a senior surgeon in the group delegating a new junior to collect data, demonstrating ownership of the study at an early stage. The department engaged in consensus building at a senior surgical level around areas of discordance with guidelines. The department identified target levels of compliance of $90 \%$.

Site 2: Engagement was consistently challenging at site 2 . The junior doctor who was initially delegated to collect data found it difficult to commit time to the study due to conflicting demands from clinical work. Another junior collected data, but this junior was not directly part of the surgical unit. Senior staff ownership was not achieved.

Site 3: Engagement at site 3 was variable throughout the study period. One junior delegated initially was effective and presented at the collaborative meeting. At that point, data collection ceased and there was no engagement for a long period. Between cycles 8 and 9 at site 3 , a critical meeting was held. Limited data were available to present but of 32 patients audited, 16 were inconsistent with clinical guidelines. Hierarchical influences, engagement in the study and reasons for discordant prescribing were extensively discussed. One senior surgeon clearly articulated 'I really want us to do better'. This provided a turning point in the meeting. Juniors were delegated to collect data. Between cycles 10 and 11 (see figure 2C), due to a persistent lack of data collection, the facilitator offered for site 3 to withdraw from the study. This resulted in a series of responses from the team at site 3 who did not wish to withdraw, engagement of a senior surgeon within the department and delegation to an enthusiastic junior doctor for data collection. Engagement was sustained for the remainder of the study.

\section{Semistructured interview arm: reflections on implementation}

At three sites, a total of 29 participants (25 doctors and 4 pharmacists) agreed to participate in semistructured qualitative interviews during August/September 2019. Nine participants were from site 1, 13 from site 2 and 7 from site 3 . Of the doctors, 11 were senior doctors (1 
Table 3 SAP discordance with clinical guidelines during preintervention and postintervention phases

\begin{tabular}{lll}
\hline $\begin{array}{l}\text { Site } \\
\text { All participants (three sites) }\end{array}$ & $\begin{array}{l}\text { Preintervention } \\
852\end{array}$ & $\begin{array}{l}\text { Postintervention } \\
905\end{array}$ \\
\hline $\begin{array}{l}\text { Correct SAP decision (all } \\
\text { aspects) }\end{array}$ & $479(56 \%)$ & $638(70.5 \%)$ \\
$\begin{array}{l}\text { SAP given when not } \\
\text { indicated }\end{array}$ & $82(9.6 \%)$ & $44(4.9 \%)$ \\
\hline Choice error & $86(10.1 \%)$ & $58(4.9 \%)$ \\
\hline Dosing error† & $243(40.3 \%)$ & $194(21.4 \%)$ \\
\hline Timing errorł & $209(24.5 \%)$ & $287(31.7 \%)$ \\
\hline Duration error§ & $50(5.9 \%)$ & $51(5.6 \%)$ \\
\hline Redosing errorף & $15(1.7 \%)$ & $14(1.7 \%)$ \\
\hline
\end{tabular}

Site 1

\begin{tabular}{lcc} 
SAP given and not indicated & $25(8.3 \%)$ & $5(1.7 \%)$ \\
\hline Choice error & $33(14 \%)$ & $26(10 \%)$ \\
Dosing error & $116(39 \%)$ & $70(24 \%)$ \\
\hline Timing error & $65(29 \%)$ & $93(35 \%)$ \\
\hline Duration error & $17(7 \%)$ & $16(6 \%)$ \\
\hline Redosing error & $3(3 \%)$ & $6(4 \%)$ \\
Site 2 & & \\
SAP given and not indicated & $44(14.7)$ & $27(9.0 \%)$ \\
Choice error & $36(13 \%)$ & $15(6 \%)$ \\
\hline Dosing error & $84(28 \%)$ & $68(23 \%)$ \\
\hline Timing error & $70(26 \%)$ & $102(38 \%)$ \\
\hline Duration error & $28(10 \%)$ & $9(3 \%)$ \\
Redosing error & $7(10 \%)$ & $2(3 \%)$ \\
Site 3 & & \\
\hline SAP given and not indicated & $13(6.6 \%)$ & $12(5.5 \%)$ \\
\hline Choice error & $17(9 \%)$ & $17(6 \%)$ \\
\hline Dosing error & $43(22 \%)$ & $56(18 \%)$ \\
\hline Timing error & $74(42 \%)$ & $92(33 \%)$ \\
\hline Duration error & $5(3 \%)$ & $26(9 \%)$ \\
\hline Redosing error & $5(7 \%)$ & $6(9 \%)$ \\
\hline P 0.05. & & \\
\hline
\end{tabular}

$\mathrm{P}<0.05$.

${ }^{*}$ Choice relates to the type of antibiotic chosen.

tDosing errors included dosing of therapeutic antibiotics that were being assessed as whether they were appropriate for SAP (if SAP was indicated and not given in addition to therapeutic antibiotics).

fTiming was incorrect if SAP was not given within the locally recommended guidelines interval before incision (predominantly $60 \mathrm{~min}$ ). If SAP was indicated and the patient was on treatment antibiotics the timing of treatment antibiotics was assessed against local guidelines for SAP adequacy. $\S M e d i c a l$ charts were reviewed to examine whether SAP was continued postoperatively and this was assessed against guidelines for duration. Most operation guidelines recommended a single dose of SAP.

IThe compliance with redosing in prolonged operations and where substantial blood loss occurred was assessed.

SAP, surgical antibiotic prophylaxis.

infectious diseases physician and 10 surgeons) and 14 were junior doctors of varying levels of experience.

The four themes were identified from the qualitative interviews as impacting on the success of the intervention: (1) the effect of senior engagement on implementation, (2) the influence of internal characteristics of the department influencing intervention uptake (adaptability, receptiveness to change), (3) the significance of
Table 4 Within-site comparison of SAP prescribing

\begin{tabular}{cccc}
\hline Site number & Result (95\% Cl) & $\mathbf{R}^{2}$ & P value \\
\hline Site 1 & & 0.97 & \\
$\beta_{\text {pre }}$ & $3.67(1.82$ to 5.52$)$ & & 0.002 \\
$\Delta$ & $-10.38(-19.75$ to -1.01$)$ & & 0.034 \\
$\beta_{\text {post }}$ & $5.62(3.77$ to 7.47$)$ & & $<0.001$ \\
Site 2 & & 0.89 & \\
$\beta_{\text {pre }}$ & $1.81(-1.24$ to 4.85$)$ & & 0.209 \\
$\Delta$ & $19.20(3.70$ to 34.69$)$ & & 0.021 \\
$\beta_{\text {post }}$ & $-3.71(-6.75$ to -0.66$)$ & & 0.023 \\
Site 3 & & 0.62 & \\
$\beta_{\text {pre }}$ & $-0.69(-5.58$ to 4.19$)$ & & 0.752 \\
$\Delta$ & $5.90(-18.94$ to 30.75$)$ & & 0.599 \\
$\beta_{\text {post }}$ & $3.78(-1.11$ to 8.67$)$ & & 0.112 \\
All sites & & 0.98 & \\
$\beta_{\text {pre }}$ & $1.51(0.234$ to 2.80$)$ & & 0.026 \\
$\Delta$ & $6.13(-0.35$ to 12.61$)$ & & 0.061 \\
\hline$\beta_{\text {post }}$ & $1.56(0.28$ to 2.84$)$ & & 0.023 \\
Overall & & 0.87 & \\
Site 1 $(\beta)$ & $3.89(0.60$ to 7.17$)$ & & 0.029 \\
Site 2 $(\beta)$ & $-1.43(-4.49$ to 1.63$)$ & & 0.283 \\
Site 3 $(\beta)$ & $4.21(2.47$ to 5.95$)$ & & 0.002 \\
\hline All sites $(\beta)$ & $2.22(1.23$ to 3.21$)$ & & 0.002 \\
\hline
\end{tabular}

SAP, surgical antibiotic prophylaxis.

guideline consensus building and guideline visibility, and (4) the power of structural barriers in limiting uptake of the intervention. See box 1 for verbatim quotes supporting key elements of these themes.

The effects of senior engagement on implementation

A significant theme within the qualitative interviews across the three sites was the identified need for senior ownership and engagement for successful implementation of the study. Although participants at all sites universally agreed that SAP was an important issue and that the study was of value to their department, there were significant barriers to engagement identified in the qualitative interviews at sites 2 and 3. Junior doctors at site 2 discussed lack of senior ownership in the department as a significant barrier to the intervention effectiveness. Although senior doctors at sites 2 and 3 were supportive of the project in theory, in practice their support was reported to be limited by a range of competing concerns and pressures. At site 2 , one barrier that was identified was a lack of clarity as to who was responsible for maintaining the momentum of the study, with senior surgeons identifying junior doctor rotations as halting study momentum, and conversely junior doctors identifying lack of senior surgeon engagement as having the same impact. At site 1 , senior engagement was uniformly reported by participants (junior and 


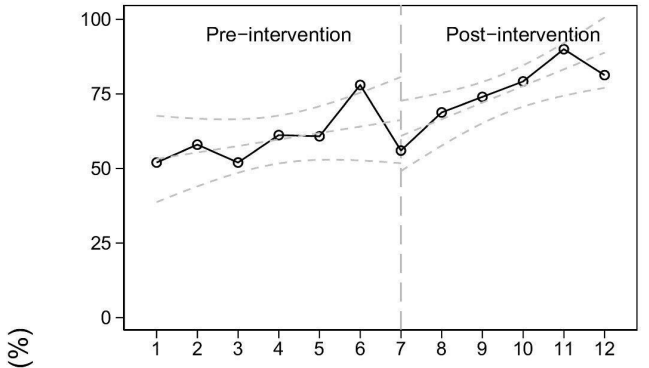

(A) Site 1

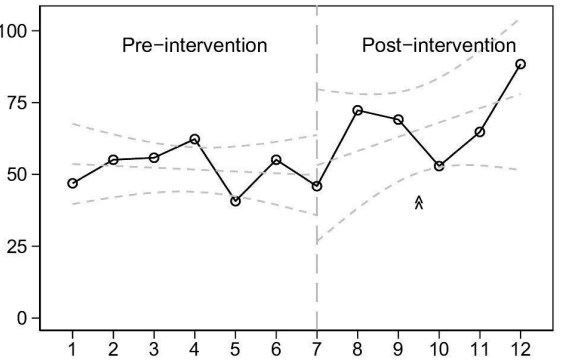

(C) Site 3

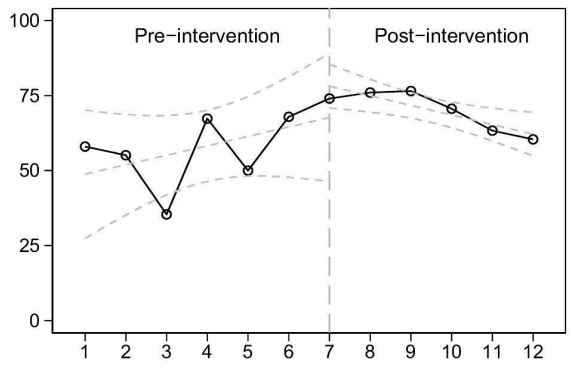

(B) Site 2

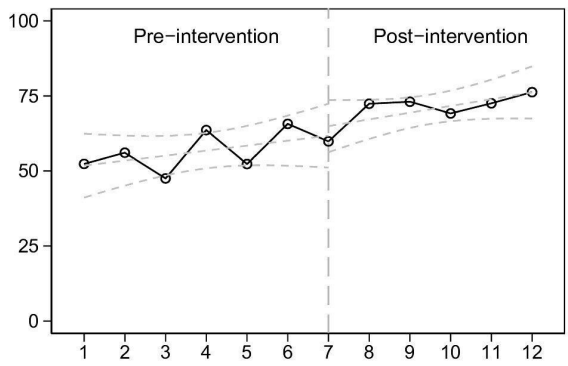

(D) All Sites

Figure 2 Compliance percentage by site over time: the sites are identified in each panel (site 1= A, site 2=B, site 3=C, and $\mathrm{D}$ represents combined data from all sites). In every case, the vertical axis represents the compliance percentage and the horizontal axis the bimonthly cycle number with the intervention occurring during cycle 7 . The point at which site 3 was offered by the facilitator to withdraw from the study is illustrated by the double caret in panel (C).

senior) and there were no identified barriers to data collection or participation in the study. At site 1, multiple participants reflected on the long-term relationship with the infectious diseases department as having a significant role in their engagement in the study.

Intervention implementation and 'Adaptability' of the department Multiple members of site 1 reflected on their department's flexibility around change processes, openness to innovation and the involvement of key opinion leaders in a departmental culture that was open to change, as significant enablers in uptake of the study. In site 2, some participants reflected on the culture of junior versus senior members of the department, identifying some older senior surgeons as being more fixed to their current practice and unwilling to change, which impacted on the momentum for change in the department. In site 3 , participants also reflected on the inability to influence senior surgeons' practice.

Visibility, uptake and producing day-to-day consensus

All sites requested guidelines on the operating theatre walls and reflected on the role of the guidelines on the wall as an enabler of prescribing compliance. Multiple participants across sites recognised the value of this in prompting guideline-based decision-making both through providing a continual and at-a-glance reminder of the guidelines and through promoting consensus around the guidelines. Facilitated discussions were also reported to help build consensus around guidelines and were reported across sites as adding value and building prescribing consistency within departments.
Structural barriers to intervention implementation

At both sites 2 and 3, there were significant reported barriers to ownership at both a junior and a senior level. Both sites 2 and 3 reported issues with staff resourcing, resulting in time pressure which required prioritisation of clinical work over quality improvement strategies. Site 2 was undergoing a significant expansion (rebuilding, moving to electronic records in theatre, etc) during the study, and as such there were multiple change processes occurring concurrently which were reported to result in change fatigue and reduced ability to focus on quality improvement strategies such as QSAP. There was internal conflict and sadness expressed about inability to provide adequate attention to each desirable change process in such an environment.

\section{DISCUSSION}

SAP prescribing is widely acknowledged to be suboptimal globally, and strategies are urgently required to improve both operative outcomes and reduce the antibiotic pressure for AMR progression. There is significant evidence regarding the social influences on prophylaxis, and these have been shown to be pervasive across many clinical contexts (ie, fear, risk, norms, habit, lack of leadership). However, there has been little innovation around addressing and/or harnessing these influences to enact change. Indeed, whether the use of such influences might provide a more powerful, sustainable or cost-effective strategy for SAP guideline compliance improvement across diverse clinical contexts. While it has 


\section{Box 1 Indicative quotes}

Theme 1: The effects of senior engagement on implementation Site 1

I think those two surgeons within the group and being on board with it probably helped quite a lot (...). If someone like him said, 'This is worthwhile and worthwhile looking at,' then I think you'd be foolish not to strongly consider it at least. (P24, senior surgeon)

I think it's received really well. I think, in part, because of the previous relationships and history between infectious diseases, and [JB] and the surgeons, I think that made them more receptive to signing up to it and doing it. I think if you'd have just come in as an outsider and said, 'I want to do this study,' everyone would have looked and said, 'Who are you and why?' People are a bit suspicious maybe. (P22, pharmacist)

I think it [satisfaction with the study] was very high for us in general surgery across all the levels of personnel. Particularly for the consultants, I think it really brought us closer to ID as well, and that really helped with other bits of our work, intensive care and all this sort of stuff. (P21, senior surgeon)

\section{Site 2}

I don't know if we could have engaged the consultants a bit more, engaged the team more, in a way. It's hard, but I would've wanted a bit more involvement from the consultants, I guess. And just a general team. But I guess you've got to start from somewhere, and I think it's brought about a start, at least. (P8, mid-level surgeon)

And, as I said, it's up to leadership sometimes, it's surgical team. Some are very keen and ready to implement these QSAP protocols. And someone that was pulled down and not particularly keen, they want to have that independence. (P1, senior surgeon)

So, we had a good enthusiastic group of [junior doctors] who were measuring very well for us last year. For a variety of reasons, the [junior doctors] who put their hands up to do the measurement in the last six months, haven't been as attentive to what they needed to do as they could have been. (P13, senior surgeon)

\section{Site 3}

...last year we had some of those keen on collecting data. So, they collect all the data, most of the data, and then when they left, the data collection just dropped a bit, or dropped significantly because, although people are keen to do it, it's just they just don't have the time. (P14, senior surgeon)

So, if the consultants are on board, things will change or will happen. If they're not on board, then it will never work basically. But then how do you get the consultants on board? (P16, mid-level surgeon) It's very well received. I think this is something that we need to do. And yeah, I think it's been very well received. And Jennifer presented her findings in her talk in our meeting. It was very well received, and we're all very supportive. We need to do that. (P17, senior surgeon)

Theme 2: Intervention implementation and 'Adaptability' of the department

\section{Site 1}

I think from our departmental point of view, our department is quite receptive in changing practices that are deemed for the collective good of the patient... I mean, it just depends on experience, age, what you're used to using, what evidence you've had in the past for it. So, I think we're quite younger and progressive, so we're happy to take on board changes. (P23, senior surgeon)
Box 1 Continued

I think [the culture is] reasonably good. So over the last eight, 10 years, research has become an increasingly accepted part of business. (P27, senior surgeon)

I think our team is pretty good and we are not really rigid. We are flexible people. And when we have the right, but we don't believe without evidence, but if there's right information, we always happy to change. That's what I have done. I know most of our surgeons have changed, especially with [Facilitator's name], these updates and lectures and the heads up and her audits made us. (P20, senior surgeon)

\section{Site 2}

I guess, mixed [reception]. Obviously people were interested in it to see how compliant and concordant the department was in the guidelines. Sometimes there was interesting discussions surrounding certain findings where they weren't entirely compliant, but still open to discussion and the possibility of change... I mean, there is always a little bit of it [pushback]. Whenever there's change, there's always a bit of scepticism... (P9, junior doctor)

I just think generally when someone tells you what to do, it can just rub you up the wrong way. But I think that's human nature. So, I suppose, just a personality trait that some people are really positive about everything, some people just take a while to agree to things. (P2, pharmacist)

... I think initially at least, people didn't take it seriously. I guess, as with anything, people would just sort of brush it off and go, 'Yeah, just something else.' When people don't respond to those guidelines, they don't understand why certain things are done in certain ways, I guess people don't respond to it. (P8, junior doctor)

\section{Site 3}

...sometimes we do things just according to our own preference. Yeah, that is true. I think we all try not to do that. But, I guess, that is the aspect that I think is the weakest part of us. (P17, senior surgeon)

It's the people that are already at the end who have done this for the same way for 15 years that don't want to change it. So, long-term I think it will work. It's just that in the short-term it's difficult because of the bosses. But I don't really know how to get them to change their ways unfortunately. (P16, mid-level surgeon)

\section{Theme 3: Visibility, uptake and producing day to day consensus} Site 1

In terms of all antibiotic prophylaxis now, we've got access to the big posters in all theatres now. And I would have to say that guides me in terms of what antibiotic prophylaxis I use routinely. In addition to that, [facilitator] talked to us on multiple occasions and highlighted some of the deficiencies which may not been previously thought about. (P26, senior surgeon) I think there's been a much more sort of consensus approach to antibiotic prophylaxis that's utilised now. A lot are due to the efforts from Jennifer Broom in terms of highlighting what is standard practice. And then, I guess, probably some of those small outliers would probably bring their practice back into line with what we're doing as a group... (P26, senior surgeon) 


\section{Box 1 Continued}

\section{Site 2}

I think what's also very helpful is the posters in the washing sink, just to remind us. Sometimes we do forget, and when we're washing our hands we'll be like, 'Okay, yes, this is what operation we're doing and this is what antibiotic we should be giving.' (P10, midlevel surgeon)

I think [QSAP] opened a channel of discussion to make people who are involved in antibiotic prophylaxis, make them think and become more aware of the guidelines.... It promoted knowledge. (P9, junior doctor)

\section{Site 3}

Well, the guidelines, sort of having the guidelines put up in the wash area, like where the-it's quite good because you always look at that to see what it is. I'm here as well as the [Other hospital name] public, as well as private. They put a think in the sinks, the wash basins, that say, 'There's prophylaxis that you need to do. This is the guidelines.' (P14, senior surgeon)

\section{Theme 4: Structural barriers to intervention implementation Site 2}

And so, the QSAP thing on its own, you need someone driving it. And in the context of a place like [site 2], which has gone through an implosion and is rebuilding, there are meetings all the time for all sorts of things, [...]There's constant quality meetings, vision meetings, all these things going on all the time. So, people can get fatigue, survey fatigue, data fatigue. (P7, senior infectious diseases physician) And particularly now, we're now moving from this paper-based files to electronic one, which you don't always have access at that moment. So, that might lead sometimes to some mistakes. (P1, senior surgeon)

\section{Site 3}

Our unit is very busy, and so people don't have the time to do things like that [auditing], which is quite sad, but that is what has happened. [...] ... probably it's contribute maybe a lower socioeconomic sort of demographic means that the place is always busy, that often doctors that work for us often don't have time to collect the data. Like, they would be on-call more, all of the time, most of the time, but they just didn't have the time to get the data. (P14, senior surgeon)

been clear that a new model of SAP intervention (and indeed AMS itself) is required, there has been hitherto a lack of capacity to integrate our understandings of why (suboptimal prescribing continues) with how (to meaningfully implement AMS within SAP).

Given this, we embarked on the development, deployment and assessment of a novel intervention that focused on a three-tiered strategy, centred on a barriers-enablersownership model, which was derived from evidence of prevailing influences both nationally and internationally. This strategy sought to integrate real-time, localised audit data, with deliberative, ground-up group sessions whereby barriers to change could be discussed and challenged. The intervention itself, as expected, was differentially experienced and improvements were varied across sites, and varied within sites over time. As audit feedback and discussions illustrated change, sites changed their approach (re-engagement as seen in site 3, or stepping back even further, as in site 2).

While the barriers-enablers-ownership model, pursued here, was associated with ongoing consistent improvement in SAP in site 1, and in the later stage of site 3, the greater lessons here are identified from the situations when it did not receive significant uptake or whereby the site did not see a significant improvement in SAP (site 2 ). There were clear signs during the study (lack of data collection, lack of senior ownership) in site 2 and early on in site 3 that ownership was not occurring. When paired with the qualitative data, the identified factors include resource limitations, conflicted priorities and lack of understanding as to who was required to 'own' the study. Building an assessment of engagement/ownership into any AMS intervention and reframing the intervention (or addressing barriers) at a site if engagement is not present may be key to future study designs. This might include both a preintervention assessment as to a department's openness and willingness to engage in change processes (based on simple measures such as: do the senior members of the department schedule meetings to discuss the intervention, do they participate in discussion and design of the intervention and do they delegate resources within their department such as juniors to contribute to the quality improvement process). If engagement is not present, consideration of the wider priorities/resources/ limits of the organisational context is likely to be more effective than persisting in implementation. An approach which acknowledges the very real limiting factors that clinical leaders face in the conflicts of their day-to-day priorities may in the longer term enable enduring relationships and potentially a change in prescribing. For example, site 2 continues to engage with investigators after study to look at internal changes which may facilitate improvement in prescribing. The longer term embedded AMS programme at site 1, which focused on engagement rather than restriction, may have had a substantial impact on the willingness of site 1 to engage in the study, and indeed on the improvements in SAP prescribing which were beginning prior to study commencement, even though previous interventions had not been focused on SAP prescribing specifically. ${ }^{28}$

The increase in compliance, seen in this study, after an offer to withdraw from the study was made to site 3 , could indicate a potential behaviour change technique to address barriers aligned with social influences. The literature on guideline implementation indicates that multiple strategies are needed to address the variety of factors that can impede compliance. ${ }^{34}$ This appeal to the competitive nature of senior leadership at the site may have led to reconsideration of the advisability of withdrawal. This effect is commonly seen in studies of benchmarking. ${ }^{35}$ The literature on benchmarking also supports the way in which the offer to withdraw was made. A punitive approach would have been alienating to the team. 
Recognition of the barriers to engagement was part of the communication and allowed the team to reconsider the resources they brought to the project.

The barriers-enablers-ownership model implemented here showed considerable value across the sites, but the study also illustrated enduring challenges which were illuminated via the qualitative descriptive analysis. This descriptive phase provides additional data on why various improvements were not seen across site, and furthermore, what is required in the future in addition to the current intervention. These interviews, taking place at the latter stages of the intervention phase, illustrated the ongoing challenges of senior buy-in (leadership), structural vulnerabilities (institutional change), maintaining attention on the problem (day-to-day visibility), as well as general characteristics of the local environment (adaptivity and openness to change). Such data on the complexity of implementation should be considered when designing the roll-out of SAP optimisation strategies and stewardship more broadly. There is well-recognised urgent need for evidence around what facilitates successful implementation of stewardship practices, ${ }^{36}$ and this study begins to provide critical insights into what embedded stewardship requires.

The limitations of this study include the implementation in one state within Australia, and although such influences on prescribing are likely to be transferable to other locations both nationally and internationally, further study of the barriers-enablers-ownership approach in diverse contexts and locations would be of value.

\section{CONCLUSIONS}

A new model of AMS implementation in the context of SAP is demonstrated, focusing on barriers-enablersownership, and this model has the potential to integrate stewardship within departments (and likely to have relevance beyond surgical specialties), driven by senior clinical leaders within specialties. A dynamic facilitated process within individual units, with assessment of engagement and senior clinical ownership during an intervention, as a quality indicator for the likely success of the AMS strategy, provides an AMS model which is more sophisticated, culturally sensitive and potentially may result in the embedding of AMS principles within specialty units.

\footnotetext{
Author affiliations

${ }^{1}$ Sunshine Coast Health Institute, Birtinya, Queensland, Australia

${ }^{2}$ School of Medicine, University of Queensland, Brisbane, Queensland, Australia

${ }^{3}$ School of Social and Political Sciences, University of Sydney, Sydney, New South Wales, Australia

${ }^{4}$ School of Medicine, Griffith University, Gold Coast, Queensland, Australia ${ }^{5}$ Infectious Diseases Service, Sunshine Coast University Hospital, Sunshine Coast, Queensland, Australia

${ }^{6}$ Department of Surgery, QEII Jubilee Hospital, Acacia Ridge, Queensland, Australia

${ }^{7}$ The University of Queensland Centre for Clinical Research, Herston, Queensland, Australia

${ }^{8}$ Infection Management Service, Princess Alexandra Hospital, Woolloongabba,

Queensland, Australia

${ }^{9}$ Caboolture Hospital, Caboolture, Queensland, Australia
}

${ }^{10}$ Southern Cross University, Lismore, New South Wales, Australia

Twitter Jennifer Broom @jenniferkbroom and Katherine Kenny @kennykatherine

Acknowledgements The work of Dr Stefanie Plage in performing the individual in-depth interviews is acknowledged. The support of local AMS teams including Mandy Ng, David Looke, Patricia Kilfoyle, Sarah Kingscote, Kathryn Wilks and Paul Chapman is acknowledged and appreciated. The support of Gareth Beall in providing expert review of guideline decisions is appreciated.

Contributors JB conceived and designed the study, facilitated the study, acquired and analysed the data, drafted and revised the manuscript and approved the final version. $A B, C A$ and $M C W$ contributed to the study design, analysed the data, revised the manuscript drafts and approved the final version. KK, SY, DG, DS, HH and RP contributed to data analysis and interpretation, revised the drafts of the manuscript and approved the final version. AH contributed to the study design, revised the drafts of the manuscript and approved the final version. AJ, AM, RT, DF, SEP, TC, MA, LW, TLB and CL collected the data for the study, revised the drafts of the manuscript and approved the final version.

Funding This project was supported by an Advance Queensland Senior Research Fellowship and an ARC Linkage grant LP170100300.

Competing interests None declared.

Patient consent for publication Not required.

Ethics approval Ethical approval was granted by the Prince Charles Hospital Human Research Ethics Committee (HREC/18/QPCH/45).

Provenance and peer review Not commissioned; externally peer reviewed.

Data availability statement Data are available upon reasonable request.

Supplemental material This content has been supplied by the author(s). It has not been vetted by BMJ Publishing Group Limited (BMJ) and may not have been peer-reviewed. Any opinions or recommendations discussed are solely those of the author(s) and are not endorsed by BMJ. BMJ disclaims all liability and responsibility arising from any reliance placed on the content. Where the content includes any translated material, BMJ does not warrant the accuracy and reliability of the translations (including but not limited to local regulations, clinical guidelines, terminology, drug names and drug dosages), and is not responsible for any error and/or omissions arising from translation and adaptation or otherwise.

Open access This is an open access article distributed in accordance with the Creative Commons Attribution Non Commercial (CC BY-NC 4.0) license, which permits others to distribute, remix, adapt, build upon this work non-commercially, and license their derivative works on different terms, provided the original work is properly cited, appropriate credit is given, any changes made indicated, and the use is non-commercial. See: http://creativecommons.org/licenses/by-nc/4.0/.

\section{ORCID iDs}

Jennifer Broom http://orcid.org/0000-0003-3513-3412

Katherine Kenny http://orcid.org/0000-0001-9088-5671

Marianne C Wallis http://orcid.org/0000-0003-2753-1066

\section{REFERENCES}

1 O'Neill J. Tackling drug-resistant infections globally: final report and recommendations 2016.

2 Pierce J, Apisarnthanarak A, Schellack N, et al. Global antimicrobial stewardship with a focus on low- and middle-income countries. Int $J$ Infect Dis 2020.

3 ACSQHC. Australian Commission on safety and quality in health care. Surgical National Antimicrobial Prescribing Survey: Results of the 2016 Pilot, 2017

4 van Kasteren MEE, Mannien J, Kullberg B-J, et al. Quality improvement of surgical prophylaxis in Dutch hospitals: evaluation of a multi-site intervention by time series analysis. J Antimicrob Chemother 2005;56:1094-102.

5 Tiri B, Bruzzone P, Priante G, et al. Impact of antimicrobial stewardship interventions on appropriateness of surgical antibiotic prophylaxis: how to improve. Antibiotics 2020;9.

6 Brink AJ, Messina AP, Feldman C, et al. Netcare antimicrobial stewardship study A. from guidelines to practice: a pharmacistdriven prospective audit and feedback improvement model for peri-operative antibiotic prophylaxis in 34 South African hospitals. $J$ Antimicrob Chemother 2017;72:1227-34. 
7 Riggi G, Castillo M, Fernandez M, et al. Improving compliance with timely intraoperative redosing of antimicrobials in surgical prophylaxis. Infect Control Hosp Epidemiol 2014;35:1236-40.

8 Kim ES, Park S-W, Lee C-S, et al. Impact of a national Hospital evaluation program using clinical performance indicators on the use of surgical antibiotic prophylaxis in Korea. Int $\mathrm{J}$ Infect Dis 2012;16:e187-92.

9 Tourmousoglou CE, Yiannakopoulou EC, Kalapothaki V, et al. Adherence to guidelines for antibiotic prophylaxis in general surgery: a critical appraisal. J Antimicrob Chemother 2008;61:214-8.

10 de Jonge SW, Gans SL, Atema JJ, et al. Timing of preoperative antibiotic prophylaxis in 54,552 patients and the risk of surgical site infection: a systematic review and meta-analysis. Medicine 2017;96:e6903.

11 Bull AL, Worth LJ, Spelman T, et al. Antibiotic prescribing practices for prevention of surgical site infections in Australia: increased uptake of national guidelines after surveillance and reporting and impact on infection rates. Surg Infect 2017;18:834-40.

12 Gagliardi AR, Fenech D, Eskicioglu C, et al. Factors influencing antibiotic prophylaxis for surgical site infection prevention in general surgery: a review of the literature. Can J Surg 2009;52:481-9.

13 Brink AJ, Messina AP, Feldman C, et al. Netcare antimicrobial stewardship study A. from guidelines to practice: a pharmacistdriven prospective audit and feedback improvement model for peri-operative antibiotic prophylaxis in 34 South African hospitals. $J$ Antimicrob Chemother 2016.

14 Duane TM, Zuo JX, Wolfe LG, et al. Surgeons do not listen: evaluation of compliance with antimicrobial stewardship program recommendations. Am Surg 2013;79:1269-72.

15 Çakmakçi M. Antibiotic stewardship programmes and the surgeon's role. Journal of Hospital Infection 2015;89:264-6.

16 Sartelli M, Labricciosa FM, Barbadoro P, et al. The global alliance for infections in surgery: defining a model for antimicrobial stewardship-results from an international cross-sectional survey. World J Emerg Surg 2017;12:34.

17 Broom J, Broom A, Kirby E, et al. How do Hospital respiratory clinicians perceive antimicrobial stewardship (ams)? A qualitative study highlighting barriers to AMS in respiratory medicine. J Hosp Infect 2017.

18 Broom J, Broom A, Plage S, et al. Barriers to uptake of antimicrobial advice in a UK Hospital: a qualitative study. J Hosp Infect 2016;93:418-22.

19 Broom JK, Broom AF, Kirby ER, et al. How do professional relationships influence surgical antibiotic prophylaxis decision making? A qualitative study. Am J Infect Control 2017.

20 Broom J, Broom A, Kirby E, et al. Improvisation versus guideline concordance in surgical antibiotic prophylaxis: a qualitative study. Infection 2018;46:541-8.
21 lerano C, Thursky K, Peel T, et al. Influences on surgical antimicrobial prophylaxis decision making by surgical craft groups, anaesthetists, pharmacists and nurses in public and private hospitals. PLoS One 2019;14:e0225011.

22 Charani E, Tarrant C, Moorthy K, et al. Understanding antibiotic decision making in surgery - a qualitative analysis. Clinical Microbiology and Infection 2017;23:752-60.

23 Baker R, Camosso-Stefinovic J, Gillies C, et al. Tailored interventions to address determinants of practice. Cochrane Database Syst Rev 2015;4:CD005470.

24 VP H, Barie PS, Stein SL, et al. Antibiotic regimen and the timing of prophylaxis are important for reducing surgical site infection after elective abdominal colorectal surgery. Surg Infect 2011;12:255-60.

25 TATC. SAGE handbook on mixed methods research in the behavioral \& social sciences. 2nd edn. Thousand Oaks, CA: Sage, 2010.

26 J C. Research Design. Qualitative, quantitative and mixed methods approaches. 3rd Ed.. Thousand Oaks, CA: Sage, 2009.

27 Schweizer ML, Braun BI, Milstone AM. Research methods in healthcare epidemiology and antimicrobial StewardshipQuasi-Experimental designs. Infect. Control Hosp. Epidemiol. 2016;37:1135-40.

28 Broom J, Tee C, Broom A, et al. Addressing social influences reduces antibiotic duration in complicated intra-abdominal infections. A mixed methods study. ANZ J Surg 2018.

29 Zhang F, Wagner AK, Ross-Degnan D. Simulation-Based power calculation for designing interrupted time series analyses of health policy interventions. J Clin Epidemiol 2011;64:1252-61.

30 Harvey G, Kitson A. PARIHS revisited: from heuristic to integrated framework for the successful implementation of knowledge into practice. Implementation Sci 2015;11:33

31 Pope C, Mays N. Qualitative research in health care. John Wiley \& Sons, 2013.

32 Fitzpatrick R, Boulton M. Qualitative research in health care: I. The scope and validity of methods. J Eval Clin Pract 1996;2:123-30.

33 Ezzy D. Qualitative analysis: practice and innovation. London: Routledge, 2002.

34 Solberg LI, Brekke ML, Fazio CJ, et al. Lessons from experienced guideline implementers: attend to many factors and use multiple strategies. Jt Comm J Qual Improv 2000;26:171-88.

35 Ettorchi-Tardy A, Levif M, Michel P. Benchmarking: a method for continuous quality improvement in health. Hcpol 2012;7:E101-19.

36 Hashad N, Perumal D, Stewart D, et al. Mapping Hospital antimicrobial stewardship programmes in the Gulf cooperation Council states against international standards: a systematic review. $J$ Hosp Infect 2020;106:404-18. 